CORRIGENDUM

doi:10.1038/nature 12667

\title{
Corrigendum: APOBEC3B is an enzymatic source of mutation in breast cancer
}

Michael B. Burns, Lela Lackey, Michael A. Carpenter, Anurag Rathore, Allison M. Land, Brandon Leonard, Eric W. Refsland, Delshanee Kotandeniya, Natalia Tretyakova, Jason B. Nikas, Douglas Yee, Nuri A. Temiz, Duncan E. Donohue, Rebecca M. McDougle, William L. Brown, Emily K. Law \& Reuben S. Harris

Nature 494, 366-370 (2013); doi:10.1038/nature11881

We reported a comparison of the DNA cytosine deamination context of APOBEC3B in vitro with the observed C-to-T mutation context in breast cancer (see Fig. $4 \mathrm{c}$ of the original Letter). We incorrectly stated in the Fig. $4 \mathrm{c}$ legend that the data represent all cytosines. However, this analysis focused on unmodified DNA cytosines and excluded all potentially methylatable 5' CpG motifs, which, being more prone to spontaneous deamination, might have skewed the analysis by providing a false positive signal; this was noted in the original Methods
Summary. It was drawn to our attention by Thanos Halazonetis that the Fig.4c legend could mislead other researchers if they were not to take this exclusion into account. We therefore provide an updated version of Fig. 4c (see Fig. 1 of this Corrigendum) with the full logo representation for the in vitro preferences of $\mathrm{APOBEC} 3 \mathrm{~B}$ and the C-to-T mutation context in breast cancer. This does not affect the central conclusion of our study that APOBEC3B is an enzymatic source of mutation in breast cancer.

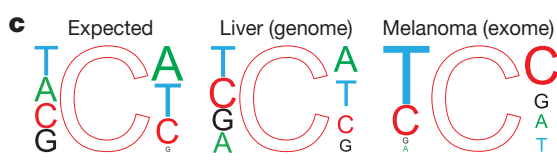

Figure $1 \mid$ This figure shows the corrected Fig. $4 \mathrm{c}$ of this Letter. c, Local sequence contexts for all genomic cytosines (expected), cytosines deaminated

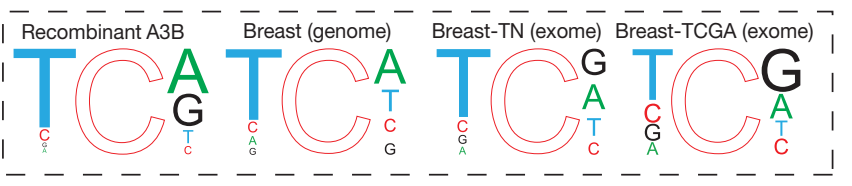

by recombinant A3B (Supplementary Fig. 13), and observed C-to-T transitions in the indicated cancers. 